\title{
Can the Sonic Radius in ADAF Be Large?
}

\author{
F. Yuan
}

Astronomy Department, Nanjing University, Nanjing 210093, P.R.China

K.L. Huang

Physics Department, Nanjing Normal University, Nanjing 210097, P.R.China

$\mathrm{Ju}-\mathrm{fu} \mathrm{Lu}$

Center for Astrophysics, University of Science and Technology of China, Hefei 230026, P.R.China

\begin{abstract}
We obtain the global transonic solutions by solving numerically a set of equations describing advection dominated accretion flows around black holes. We find that the sonic points can locate themselves at almost any radius, independent of the viscosity parameter $\alpha$ and the polytropic index $\gamma$.
\end{abstract}

Recently many authors have worked upon the global solutions for advection dominated accretion onto black holes(Chakrabarti 1996a, hereafter C96; Chen, Abramowicz \& Lasota 1997, hereafter CAL; Narayan, Kato \& Honma, hereafter NKH; Peitz \& Appl 1997, hereafter PA; Igumenshchev et al. 1998, hereafter IAN). They obtain very different results especially on the important aspect such as the locations of the sonic points. C96 find that the sonic point can locate itself at almost any radius for polytropic index $\gamma=4 / 3$ while the sonic radius $R_{s}$ is small for $\gamma=5 / 3$. Both CAL and NKH find that the sonic radius $R_{s}$ can only be as large as several gravitational radii $R_{g}$. Although IAN think $R_{s}$ can vary in a small range, its value is also within several $R_{g}$. PA's results are that $R_{s}$ is small as the viscous parameter $\alpha$ is low but it becomes very large as $\alpha$ is high.

However, all the above results have discrepancies with that in inviscid adiabatic accretion flows which have been widely studied in the past years(see Chakrabarti $1996 \mathrm{~b}$ for reviews). In that case $R_{s}$ can be almost any value , regardless of $\gamma$ and $\alpha$. The ADAF equations are exactly the same as that in adiabatic flows in the limit of weak viscosity. So we expect the value of $R_{s}$ should be similar in the two cases, i.e. $R_{s}$ can be almost any value in ADAF. In fact, it should be so since the value of $R_{s}$ should be determined by the outer boundary conditions which varies in different realistic systems.

Based upon the above considerations, we study the global solutions for advection dominated accretion flows. Equations are adopted from NKH but we don't select any boundary and don't assume any boundary conditions such as standard thin disk solutions since ADAF solutions may terminate at cer- 

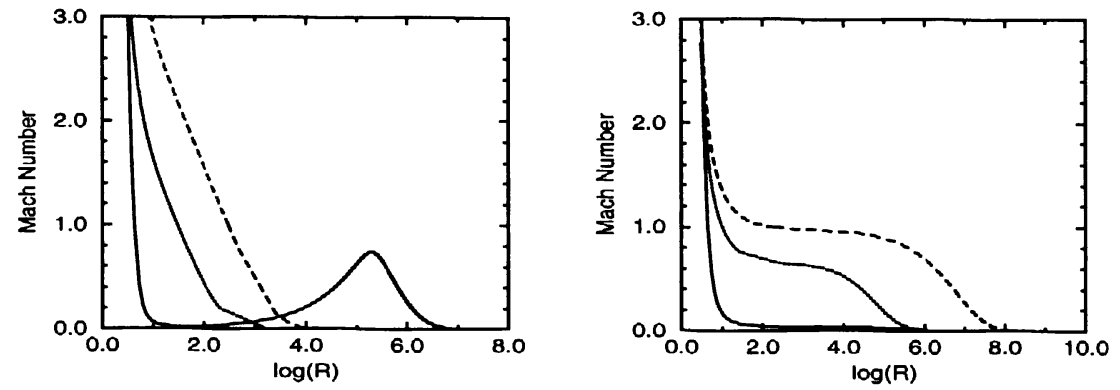

Figure 1. Left: the variations of Mach number with radius $R$ for solutions corresponding to $\alpha=10^{-3}, \gamma=1.5$ and $R_{s}=4.3, j=$ 3.696084 (solid); $R_{s}=30, j=1.75$ (dotted) and $R_{s}=300, j=$ 1.52(dashed), respectively. Right: same as the left plot, but for $\gamma=5 / 3$ , and $R_{s}=4.4, j=3.4721$ (solid), $R_{s}=10, j=2.033$ (dotted), and $R_{s}=200, j=1.54$ (dashed), respectively. $G=c=M_{\mathrm{BH}}=1$ units are used.

tain radius, and we can't assure there exist solutions under arbitrarily selected boundary conditions. We use the method presented in C96 in which two free parameters $R_{s}$ and $j$ (the angular momentum per unit mass accreted by the central black hole) are selected and the equations are directly integrated from the sonic point inward to the horizon and outward. We find that all the solutions are terminated at certain limiting radius. When we integrate outward to this radius we find that the local sound speed suddenly begins to increase steeply with $R$ which we think is unphysical. The value of the limiting radius is a function of $\alpha, R_{s}$ and $j$. What is more noticeable is that we obtain well-behaved global solutions under almost any values of parameter $R_{s}$, regardless of $\alpha$ and $\gamma$. We show some examples with $\alpha=10^{-3}, \gamma=1.5$ (left plot) and 5/3(right plot) in the figure. So our results are consistent with those in the case of inviscid accretion. This confirm our expectation.

Acknowledgments. This project was granted financial support from PanDeng Foundation.

\section{References}

Chakrabarti, S.K. 1996a, ApJ, 464, 664(C96)

Chakrabarti, S.K. 1996b, Physics Report, 266, No 5\&6, 229

Chen, X., Abramowicz, M. A., \& Lasota, J.-P. 1997, ApJ, 476, 61(CAL)

Igumenshchev, I. V., Abramowicz, M. A., \& Novikov, I. D. 1998, MNRAS, 298, 1093(IAN)

Narayan, R., Kato, S. \& Honma, F. 1997, ApJ, 476, 49(NKH)

Peitz, J. \& Appl, S. 1997, MNRAS, 286, 681(PA) 